Zum Schluss unserer Mittheilung halten wir es für unsere Pflicht, dem hochgeehrten Prof. J. R. Tarchanoff unsern Dank aussprechen für seine liebenswürdige Erlaubniss, in seinem Laboratorium arbeiten zu dürfen.

(Aus dem physiologischen Laboratorium von Prof. Tarchan of $f$ in

St. Petersburg.)

\title{
Die Bestimmung der Stromgeschwindigkeit des Blutes in den Gefässen mit dem neuen Apparat - Photo- hämotachometer.
}

Von

Dr. Napoleon Gybulski.

Hierza 13 Holzschnitte.

Obwohl in den letzten Jahrzehnten die Physiologie um zahlreiche graphische, mathematisch genaue Apparate bereichert wurde, welche uns die Lösung mancher wichtiger Fragen und Probleme ermöglichten, so blieb doch die Frage der genauen Bestimmung der Stromgesehwindigkeit des Blutes noch immer unentschieden. Um jedoch den hier sich anhäufenden Fragen etwas näher zu treten, hat man verschiedene indirecte Methoden benutzt, um wenigstens sich eine allgemeine Ansicht uiber die Frage schaffen zu können. Man benutzte z. B. zu diesem Zwecke die Bestimmung der Differenzen in der $\mathrm{Zu}$ - und Abnahme des Volumens des betreffenden Organs; man verwendete die Temperaturdifferenzen, die eintretenden Wechsel im Lumen der Gefässe, Veränderungen des seitlichen Blutdruckes in den Arterien und bestimmte auch die Stromgeschwindigkeit nach der Menge des ausfliessenden' Blutes. Alle ebenerwähnten Methoden erlauben aber nur eine qualitative Bestimmung 
der Stromgeschwindigkeit des Blutes. Ein soleher Zustand bildete bis jetzt eine nicht geringe Lücke im betreffenden Kapitel der Physiologie, um so mehr fülbar, als es Jedermann klar ist, dass die bis jetzt angewandten Apparate in jeder Beziehung unzuverlässig und zu ungenau sind.

Um diesem Uebel abzuhelfen machte ich schon seit mehreren Jahren Versuche erstens um za erfahren, auf welcher Basis der betreffende Apparat construirt werden miisste, und zweitens, in welcher Weise könnte man einen so construirten Apparat am besten ausnützen.

Zablreiche Versuche bewiesen mir, dass die Methode des "Röhrchens" von Pitot die beste sei, mit anderen Worten, dass die manometrische Bestimmung des sogenannten hydraulischen Druckes die besten Resultate liefere. Der sogenannte hydraulische Druek ist einfache Folge derjenigen Pression, welche von den Molecülen der sich in Bewegung befindlichen Flüssigkeit auf die im Wege stehenden Hindernisse ausgeübt wird.

Der Werth dieser Methode wird erbeblich dadurch gesteigert, dass das fliessende Blut in dieser Weise nur in einem selr beschränkten Maasse mit den Fremdkörpern in Berührung kommt; dieser Umstand, von einer besonderen, leider aber negativen Tragweite, tritt deutlich in allen bis jetzt angewandten Apparaten auf, weil, wie allgemein bekannt ist, die Berïhrung des Blutes mit den Apparattheilen sehr leicht die Gerinnung desselben hervorruft und die dadurch entstandenen Gerinnseln einen höchst nachtheiligen Einfluss auf die schon ungenanen Apparate ausïben.

Eine eingehendere kritische Besprechung aller bis jetzt $z \mathfrak{u}$ diesem Zwecke erfundenen Apparate will ich an dieser Stelle nicht anfihren, da sie bereits von mir anderorts gemacht worden ist. Ich werde nar kurz und bündig den von mir dazu construirten Apparat beschreiben, sowie auch in wenigen Worten tuber die trefflichen Resultate, welche ich mit ihm erhalten hatte, referiren.

Den Haupttheil des Apparates bildet ein Röhrchen, unter dem rechten Winkel gebogen mit zwei Nebenröhrehen, wie es die Fig. 1

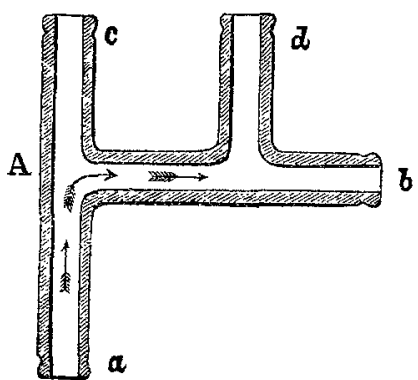

Fig. 1. 
zeigt. Wenn wir die Enden $a$ und $b$ in diesem Röhrehen mit einem anderen Röhrehen verbinden und die beiden ubrigen Enden $c$ und $d$ mit zwoi Manometern in Verbindung setzen und dann das ganze Röhrchen mit Flüssigkeit füllen, so wird uns die Flüssigkeitssäule in den beiden Manometern den gesammten Werth des Druckes zeigen, unter welchem sich die Flüssigkeit im Röhrchen befindet; wenn wir jetzt die Flïssigkeit in dem Hauptrohre $a b$ in Bewegung setzen und in der Richtung von $a$ nach $b$ treiben, wie es uns die Fig. 1 zeigt, so entsteht in beiden Nebenröhrchen $c$ und $d$ ein Unterschied in den Druckhöhen; und zwar aus dem Grunde, weil auf die Flïssigkeitssäule im Röhrchen $c$ ausser dem im ganzen Robrsystem bestehenden Seitendrucke noch diejenigen Flüssigkeitsmolecille einen bestimmten Druck ausïben, welche an der Einmündungsstelle dieses Röhrehens von ihrer primären Richtung abweichen; im Röhrchen $d$ aber steht die Fliissigkeit nur unter dem Seitendrucke. Derjenige Untersehied, welcher in der Höhe der beiden manometrischen Röhrchen entsteht, steht im gewissen strengen Verhältniss zur Stromgeschwindigkeit in dem Hauptrohre $a b$. Dieses Verhältniss muss für jede Flüssigkeit und fïr jedes Röhrchen speciell empyrisch eruirt werden. Es ist selbstverständlich, dass wenn wir schon einmal streng und genau das relative Verbältniss der Druckunterschiede in beiden Manometern zur Stromgeschwindigkeit einer gewissen Fliissigkeit bestimmt haben, so sind wir im Stande, auch umgekehrt aus der Verschiedenheit der Flüssigkeitshöhen einen Schluss über die Stromgeschwindigkeit im Rohre $a b$ zu ziehen und in solcher Weise aus der Berechnung der Niveaudifferenzen in Manometern die Stromgeschwindigkeit der Flüssigkeit im Rohre $a b$ zu berechnen.

Die Bestimmung der Abhängigkeit dieser Niveaudifferenzen in Manometern von der Stromgeschwindigkeit der Flüssigkeit im Rohre $a b$ bietet keine besonderen Schwierigkeiten dar. In meinen Untersuchungen habe ich die graphische Methode zu diesem Zweck angewandt. Zuerst bestimmte ich empyrisch auf dem experimentellen Wege die Stromgeschwindigkeit der Flüssigkeit im Rohre aus der gesammten Menge der ausfliessenden Flüssigkeit im Zeitraume von einer Secunde und zugleich notirte ich die dieser Stromgeschwindigkeit entsprechende Niveaudifferenz der Flïssigkeitssäulen in beiden manometrischen Röhrchen. Dann habe ich die Stromgeschwindigkeit in Rohre gewechselt und zwar durch 
den Querschnittwechsel der Ausflussöffnung des Rohrẹs und berechnete zugleich die Niveaudifferenz in Manometern, welche damit im Zusammenhange blieb. In solcher Weise erhielt ich eine Reihe von Werthen für die Stromgeschwindigkeit der Flüssigkeit und für die dabei entstehenden Niveaudifferenzen in den Druckböhen der beiden Manometer. Wenn ich jetzt die Werthe der manometrischen Druckdifferenzen auf die Abscisse verzeichnete und die Werthe der entsprechenden Geschwindigkeiten auf correspondirende Coordinaten verlegte, so erhielt ich für jede Fliussigkeit eine gewisse Curve, welche alsdann schon sebr leicht benutzt werden konnte zur leichten Berechnung aller anderen Stromgeschwindigkeiten derselben Flüssigkeit, sobald uns nur die Niveaudifferenzen in Manometern bekannt waren.

Die zur Prüfung der Genauigkeit dieser Methode angestellten Experimente erwiesen, dass die Febler bei der Berechnung von Stromgeschwindigkeit nach dieser Methode sehr klein sind und nicht über $1 \%$ diejenigen Zahlen übersteigen, welche wir durch die Mengenbestimmungen der ausgeflossenen Flüssigkeit erhalten. Diejenigen Hindernisse, welche wir durch die Einschaltung des gebogenen Röhrehens im ganzen Rohrsystem erzeugen, sind so geringftigig' (wie das sowohl aus rein theoretischen Betrachtungen, sowie auch aus den Untersuchungen von Volkmann und Jacobson folgt), dass wir sie nicht mehr zu berticksichtigen brauchen. Die Bedingungen, unter welchen die Bluteirculation in den Gefässen vor sich geht, wie z. B. der bedeutende Seitendruck und die Sehwankungen der Stromgeschwindigkeit, welche durch verschiedenste Momente beeinflusst werden, vor Allem aber von der Herztbätigkeit abhängig sind, gestatten uns diesen oben angeführten Gedanken auf experimentellem Wege zu prüfen. Nach mehreren misslungenen Versuchen ist mir schliesslich gelungen alle die Behinderungen, welche bei der practischen Anwendung meiner Methode zu Tage traten, glücklich zu überwinden durch die Anwendung eines differentiellen, speciell dazu construirten Luftmanometers. Der Manometer besteht aus einem gleich einer Haarnadel gebogenen Glasrobre, wie es die Zeichnung in der Fig. 2 uns veranschaulicht.

Sobald wir diesen Manometer mit den Ansatzröhrchen $c$ und $d$ an dem gebogenen Rohre $a b$ in Verbindung setzen (Fig. 1) und dann das ganze System mit der Ausnahme der höheren Hälfte 


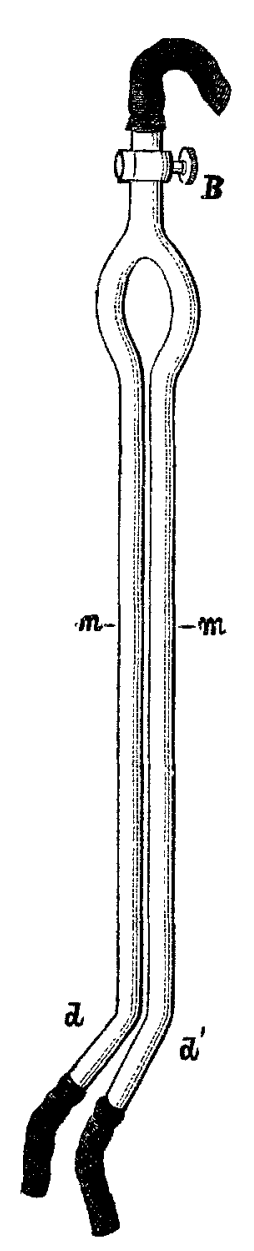

Fig. 2.

des differentiellen Manometers mit Flüssigkeit fullen und den Hahn $B$ schliessen, so stehen die beiden manometrischen Röbrehen $m$ und $m$ mit einander in Verbindung mittelst der mit Luft gefüllten Streeke im gebogenen Theile des (Luft-)Manometers. Es ist klar, dass bei jeder Druckänderung im Hauptrohre, sobald die darin befindliche Flüssigkeit sich im Zustande der Ruhe befindet, das beisst, wenn sie in keine fortschreitende Bewegung geräth, das Niveau der Flïssigkeit in beiden manometrischen Röhrchen auf einer horizontalen Linie stehen bleibt. Die Schwankungen des Seitendruckes im Röhrebensystem äussern sich nur in der gesteigerten oder verminderten Compression der Luft im oberen Theile des Manometers, was sich dadurch kund giebt, dass die beiden Niveaus gleichzeitig entweder steigen oder sinken. Wenn wir aber jetzt die Fliissigkeit im Hauptrohre $a b$ in der von Pfeilen bezeichneten Richtung sich bewegen lassen, so tritt sofort eine Niveaudifferenz in beiden manometrischen Röhrehen ein. Der Werth dieser Differenz ist gleich demjenigen Drucke, welcher von uns hydrauliseber Druck genannt worden ist und welcher stets im strengen Verhältnisse zur Stromgeschwindigkeit im Hauptrohre steht. Wir bezeichnen diesen Werth ein für alle Mal mit $h$. Wenn uns bereits der Werth von $h$ bekannt ist, so ist es gar nicht schwer, daraus die Stromgeschwindigkeit im Rohre zu bestimmen.

Beim Einsetzen des gebogenen Röbrchens in die durchgeschnittene Arterie können wir die Aenderung des Werthes $h$ leicht beobachten in dem mit dem Hauptrohre in Verbindung gesetzten differentiellen Manometer. Und wenn wir noch vordem auf empyrischem und experimentellem Wege diejenige Curve bestimmt haben, welche uns das Verhältniss von $h$ zur Stromgeschwindigkeit des Blutes bei der entsprechenden Thierart zeigt, so sind wir jetzt im Stande, aus den notirten Schwankungen des Werthes $h$ die entsprechende Stromgeschwindigkeit des Blutes zu berechnen. 
Eine solche Berechnung ist aber unmöglich aus dem Grunde, weil die Werthe $h$ einem sehr raschen Wechsel unterworfen sind. Das hat mich gezwungen eine Methode zu erfinden, welche uns die Möglichkeit giebt, das Notiren des Werthes $h$ in jedem einzelnen Augenblicke automatisch herzustellen. Bei der gewöhnlichen Stromgeschwindigkeit des Blutes in den Arterien beträgt die den Werth $h$ repräsentirende Wassersäule gewöhnlich kaum $10-30 \mathrm{~mm}$. Aus diesem Grunde ist das Anbringen aller bis jetzt bekannten, wenn auch der empfindlichsten graphischen Apparate in diesem Falle rein unmöglich, da sie alle für diese Kraft zu unempfindlich sind.

Die erfolgreiche Anwendung der Photographie bei den physiologischen Unternehmungen auf anderen Gebieten, wie es z. B. Marey gethan hatte, um die Mechanik der Bewegungen bei Thieren zu studiren, brachte mich auf den Gedanken, auch in unserem Falle für die Fixirung des Werthes $h$ Photographie anzuwenden. Zn diesem Zwecke fiel mir ein, folgende physikalische Erscheinung auszunutzen:

Eine wässerige Flüssigkeit, welche in einem engen Rohre sich befindet, benetzt in Folge der Capillarität die Wandungen der Röhre und bildet in dieser Weise eine eingesunkene Ober. fläche, wie es Fig. $3 a$ und $3 b$ zeigen.

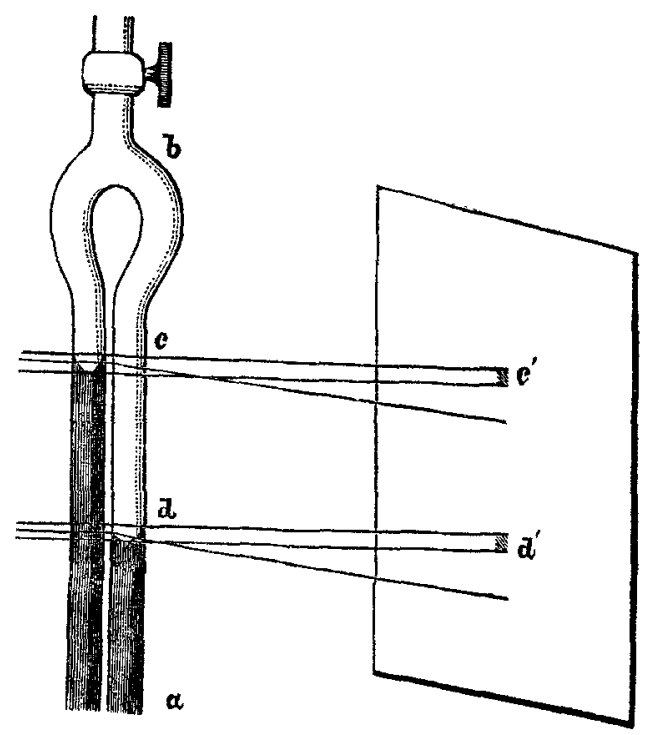

Fig. 3a. 


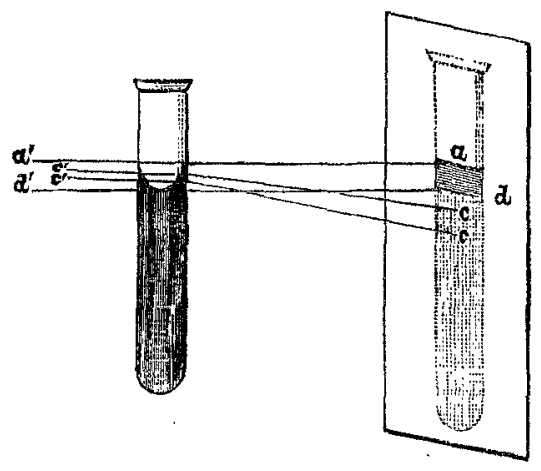

Fig. 3b.

In Folge dessen werden Lichtstralulen, welche durch das Röhrchen perpendiculär zu seiner Axe fallen, in der Riehtung zur Flissigkeit gebrochen, Fig. $3 a-c c$ und Fig. $3 b-c^{\prime \prime}-d^{\prime \prime}$, so dass wir hinter dem Röhrehen einen dunklen kleinen Streifen $a-d$ und $c-d$ erhalten, weil er der Lichtstrahlen entbehrt. Aehnliche Streifen werden erhalten auch hinter den beiden Röhrohen des differentiellen Mànometers, Fig. 3b, wo-

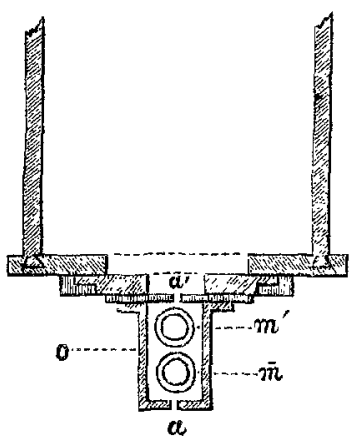

Fig. 4. bei sie selbstverständlich genau dem Niveau der Flüssigkeitssäule in jedem Röhrchen entspreehen. Zum Zweck der genauesten Bestimmung der Niveaudifferenzen der Flüssigkeiten in beiden manometrischen Röhrehen schloss ich den Manometer in eine besondere metallische Unfassung ein, welche an zwei gegenïberliegenden (entsprechenden) Seiten mit einer $1 / 2 \mathrm{~mm}$ breiten Spalte versehen war. Fig. 4 stellt uns den Manometer mit Umfassung dar.

Bei solcher Einstellung des Manometers gehen die durch die Spalte $a$ eindringenden Lichtstrahlen in der Richtung von $a$ zu $a^{*}$ durch beide manometrische Röhrehen gleichzeitig hindurch. Also wenn wir jetzt die Spaite vor dem Lichte betrachten und wenn der Manometer die Fliissigkeit enthält, so sehen wir einen dunklen sehmalen Streifen, wenn die Flissigkeit in beiden Manometern in einem Niveau steht, und $z$ we $i$ dunkle Streifen, wenn das Niveau in den manometrischen Röbrehen verschieden hoch steht. Wenn 
wir hinter den ganzen Apparat einen Spiegel stellen, so bekommen wir gleichfalls einen oder zwei dunkle Streifen, welche die helle der Spalte entsprechende Linie kreuzen.

Um die photographische Abbildung aufzufangen, habe ich den Manometer sammt Umfassung mit doppelter dunkler Kammer verbunden, wie es die Figur 5 zeigt.

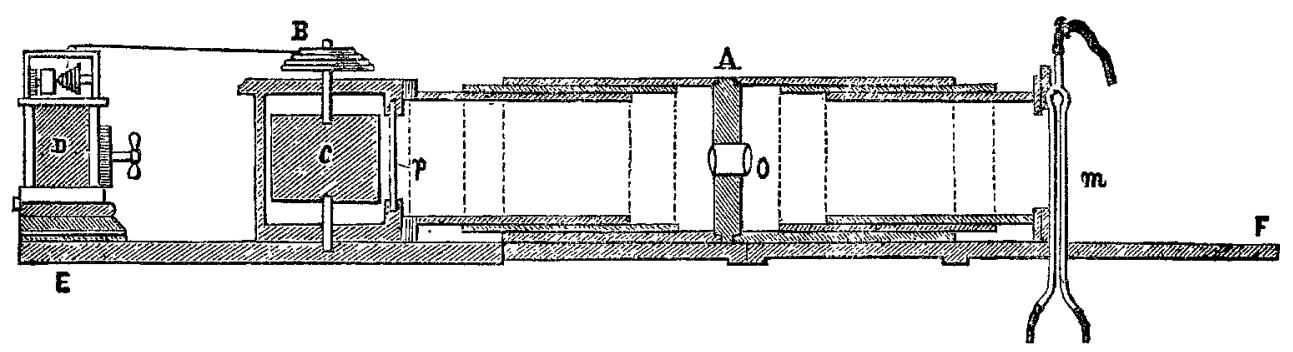

Fig. 5.

In der Wand, welche die Kammer in zwei Theile theilt, befindet sich die Linse 0 . Das Licht dringt in die Kammer ein nur durch die Spalte im manometrischen Kästchen. Jenseits der Linse bekommen wir das reelle Bild mit allen seinen Einzelheiten. Wenn wir in der Ebene des Linsenfocus ein mattes Glas einstellen und es mit einem Tuche, wie es beim Photographen gemacht wird, bedecken, so bemerken wir das reelle Bild der Spalte in Form einer hellen Linie. Wenn die beiden Oberflächen der Flüssigkeitssäulen in beiden Manometern im gleichen Niveau stehen, so wird diese Linie von einem schmalen, dunkeln Streifen quer durchschnitten. Wenn aber die Niveaus verschieden sind, so bekommen wir zwei dunkle Streifen.

Wenn aber die Entfernung der Manometer zur Objectivlinse dermaassen geregelt wird, dass die Grösse des Bildes vollkommen der Grösse des Objectes entspricht, so ist es selbstverständlich, dass in solchem Falle die Entfernung der dunkeln Streifen der wirklichen Differenz der Niveaus in beiden manometrischen Röhrchen entspricht (Fig. $3 \mathrm{~b}$ ). Um eine dauerhafte photographische Abbildung aller kleinsten Wechsel zu erhalten, muss man an der Stelle des matten Glases eine dunkle Kammer mit dem Cylinder, welcher mit dem lichtempfindlichen Bromgelatin-Papier bedeckt ist, einstellen (Fig. 5 c). Ein solcher Cylinder kann selbstverständlich mit der beliebigen Geschwindigkeit um seine veritable 
Axe rotirt werden. Der ganze Apparat muss mit grösster Sorgfalt gearbeitet werden, so dass die Lichtstrahlen ausschliesslich nur durch die Spalte in der manometrischen Umfassung eindringen können. In soleher Weise bekommen wir die photographische Abbildung von zweien Flüssigkeitsniveaus in beiden Manometern in der Gestalt von zwei weissen Linien, an den übrigen Stellen erhalten wir einen dunklen Streifen. - Die Entfernung in jedem gegebenen Augenblicke zwischen diesen beiden hellen Linien gibt uns das richtigste Maass von der wirklichen Distanz zwischen den Flüssigkeitsoberflächen in beiden Manometern. Ausserdem aber ermöglichen gewisse optische Gesetze, uns noch eine in diesem Falle sehr wichtige Erscheinung sich gelten zu lassen. Nämlich die Strecke zwischen diesen beiden hellen Streifen tritt auf dem photographischen Bilde durch ihre dunklere Färbung hervor und zwar in Folge der helleren Beleuchtung im Vergleiche zu anderen Strecken derselben Spalte. Mit Hülfe dieser Methode ist es mir gelungen, die photographische Abbildung des Werthes $h$ zu erhalten, trotzdem dass der Werth im Zeitraume einer Secunde viel wechselt.

Es ist aber von eminenter Wichtigkeit, dass man aus diesen in solcher Weise notirten Werthen $h$ leicht die ihnen entsprechende Stromgeschwindigkeit des Blutes berechnen kann und zwar in Folge des strengen Zusammenhanges der Grösse $h$ mit der Stromgeschwindigkeit der Flüssigkeit im Rohre $a b$.

Eine ganze Reihe von Untersuchungen, welche ich mit diesem Apparat ausfïhrte, hat mich zu der Ueberzeugung gebracht, dass wir in dieser Weise in den Stand gesetzt sind, alle, wenn auch die geringsten Schwankungen der Stromgeschwindigkeit des Blntes sehr genau zu bestimmen, so z. B. die Stromsehwankungen zur Zeit einer einzigen Herzevolution, dann auch bei der Systole resp. Diastole, die Schwankungen, welche mit der Respiration im $\mathrm{Za}$ sammenhange stehen, dann die Schwankungen der Blutgeschwindigkeit in den Gefässen bei Aenderungen der Körperlage, die Veränderangen der Geschwindigkeit nach dem Durehschneiden verschiedener Nerven, nach der Application von Chloroform, Curare u. s. w.

Ausserdem verdient besonders hervorgehoben zu werden der Umstand, dass wir zu gleicher Zeit anf diesem photographischen Papierstreifen auch die Curven der Athmung, des Pulses, cardiographische Curven, sowie auch die Curven des Seitendruckes und manometrische Curven photographiren können. 
Die Bestimmung der Stromgeschwindigkeit des Bilutes in den Gefässen etc. $\$ 91$

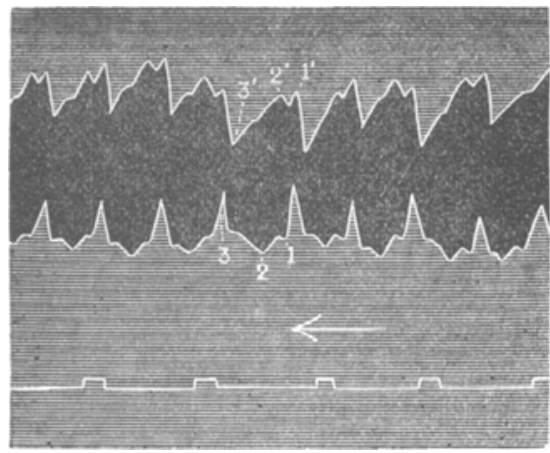

Fig. 6.

Die erhaltenen Photogramme der Niveaudifferenzen im Manometer bei Bestimmung der Schnelligkeit in A. Carotis. Die Grösse dieses Unterschiedes ist doppelt verkleinert; bei $1-1^{\prime}$ ist die Schnelligkeit gleich $248 \mathrm{~mm}$; bei $2-2^{\prime}$ gleichfalls $248 \mathrm{~mm}$; bei $3-3^{\prime}=127 \mathrm{~mm}$. Die erste Schnelligkeit entspricht der Systole, die zweite der dicrotischen Welle, die dritte der Diastole.

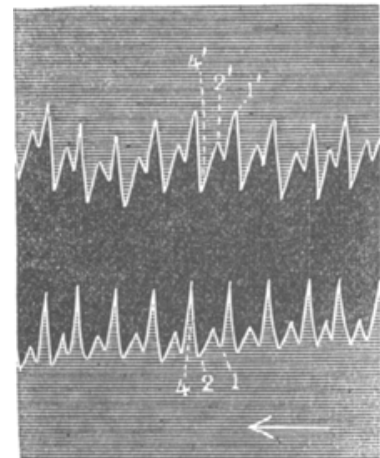

Fig. 7 .

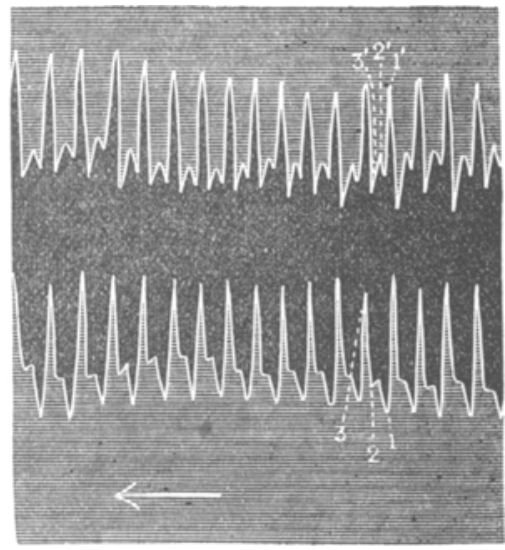

Fig. 8 .

Fig. 7. Das siebente Photogramm von demselben Thiere. Gleichfalls in A. Carotis. Die Schnelligkeit ist gleich bei $1-1^{\prime} 238 \mathrm{~mm}$, bei $2-2^{\prime}=225$, bei $3-3^{\prime}=156$. - (Die Grösse der Differenzen ist hier normal.)

Fig. 8. Die von demselben Thier erhaltenen Photogramme in A.Femoralis. Die Grösse der Differenzen ist normal. Bei $1-1^{\prime}$ ist die Schnelligkeit gleich $356 \mathrm{~mm}$, bei $2-2^{4}=300$, bei $3-3^{4}=177 \mathrm{~mm}$. 


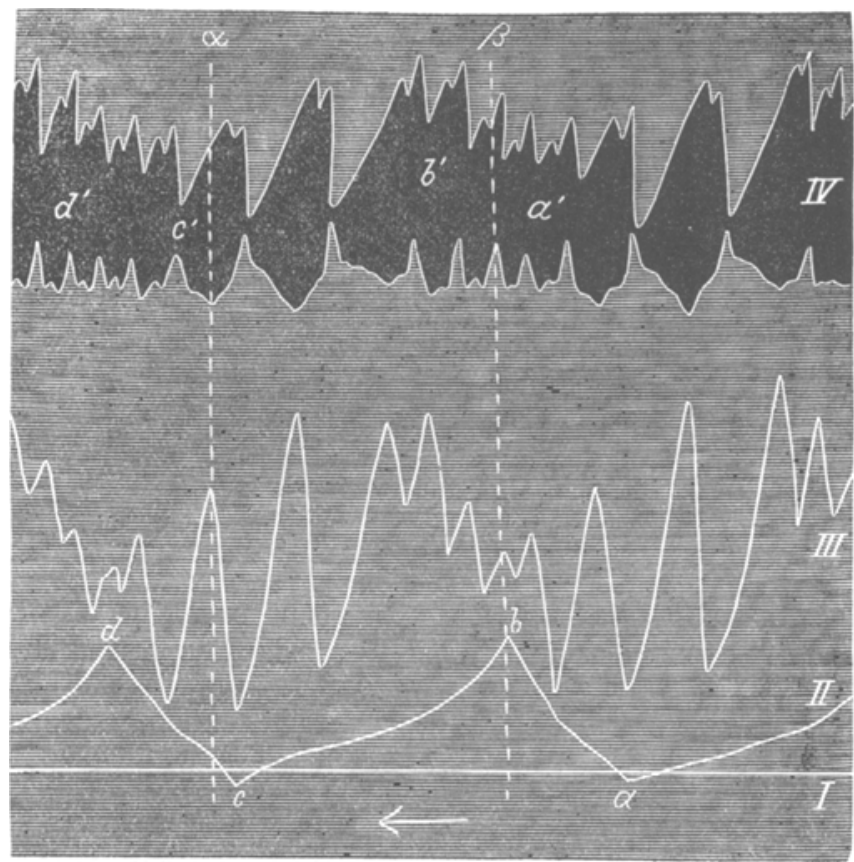

Fig. 9.

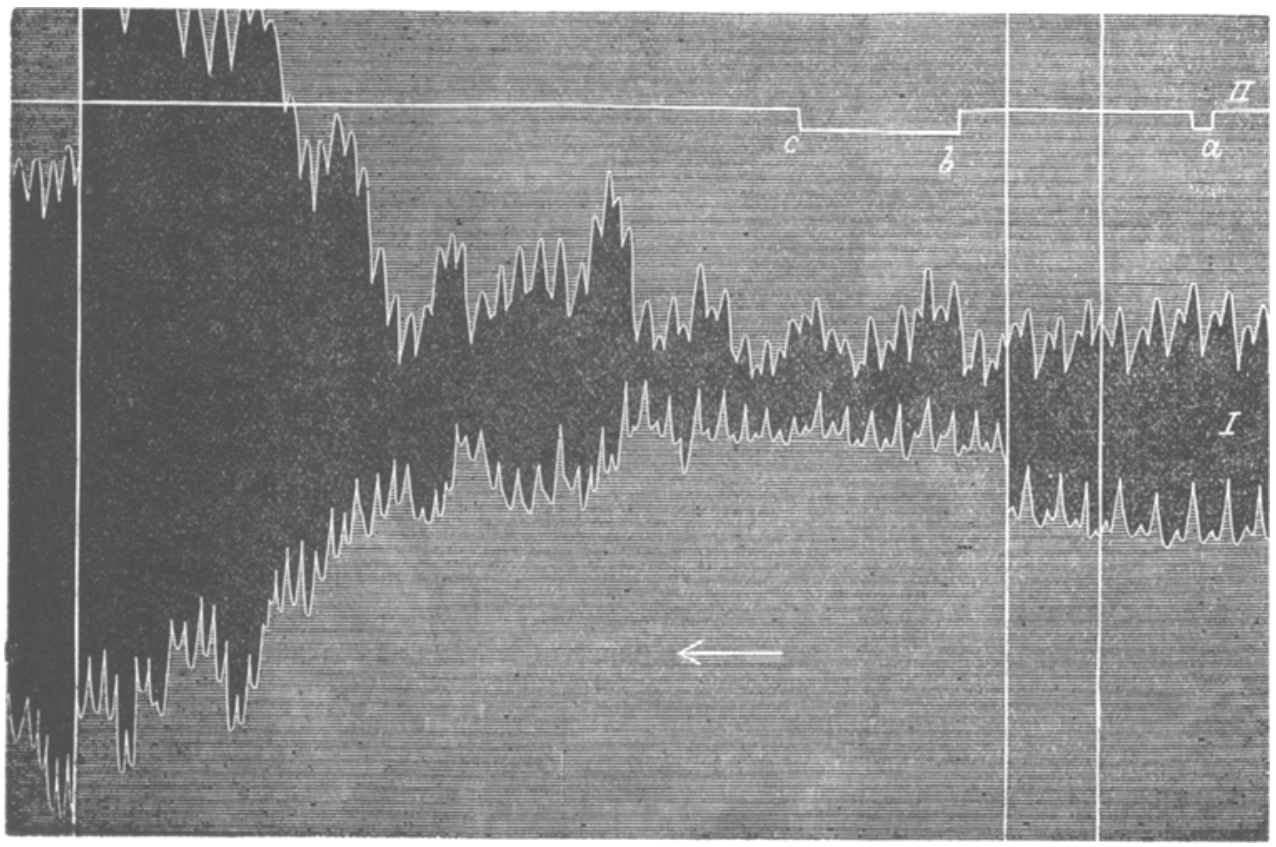

Fig. 10. 
Die Bestimmung der Stromgeschwindigkeit des Blutes in den Gefässen etc. 393

Fig. 9. In diesem Photogramme stellt I eine Linie vor, die von der Nulllinie des Manometers $70 \mathrm{~mm}$ entfernt ist; II die Curve des Athmens: $a b$ inspiratio und $b c$ exspiratio; III die Curve des Blutdruckes, Maximum $212 \mathrm{~mm}$ der Quecksilbersäule, Minimum $152 \mathrm{~mm}$; IV die Schnelligkeit, bei a` die mittlere Quantität des durchfliessenden Blutes $=2156 \mathrm{cmm}$, bei $b^{\prime}=2600 \mathrm{cmm}$.

Fig. 10. Die Untersuchung in A. Carotis: I die Schnelligkeit, II das Signal. Bei $a$ Durchschneidung des $N$. Vagus auf der entgegengesetzten Seite; bei $b$ auf derselben Seite. Die Nervi sympathici sind durchgeschnitten. Die Querlinien bezeichnen eine Pause von fünf Secunden. Die Schnelligkeit vor der Durchschneidung: Systole $238 \mathrm{~mm}$, Diastole $177 \mathrm{~mm}$. Die Schnelligkeit nach der Durchschseidung: Systole $440 \mathrm{~mm}$, Diastole $425 \mathrm{~mm}$.

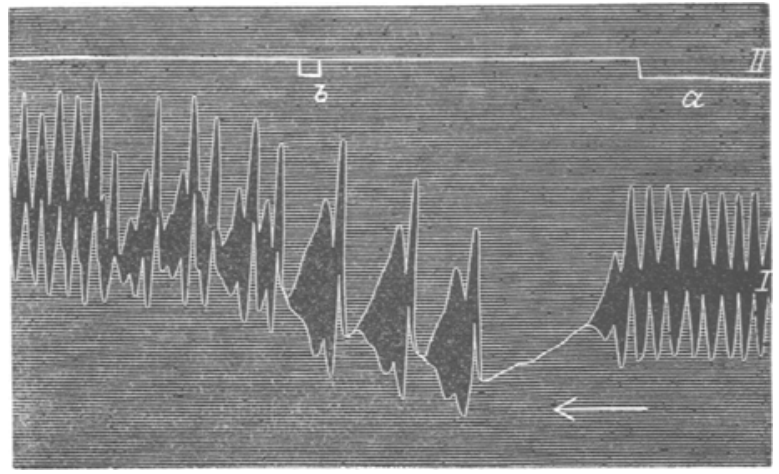

Fig. 12.

Die Photogramme der Schnelligkeit in Art. femoralis bei einem mittelst Curare vergifteten Thiere, Bei $a$ fängt die Reizung des Herzendes der $N$. vagus an, bei $b$ hört die Reizung auf.

Fig. 11 siehe umstehend. 
394 Napoleon Cybulski: Die Bestimmung der Stromgeschwirligkeit etc.

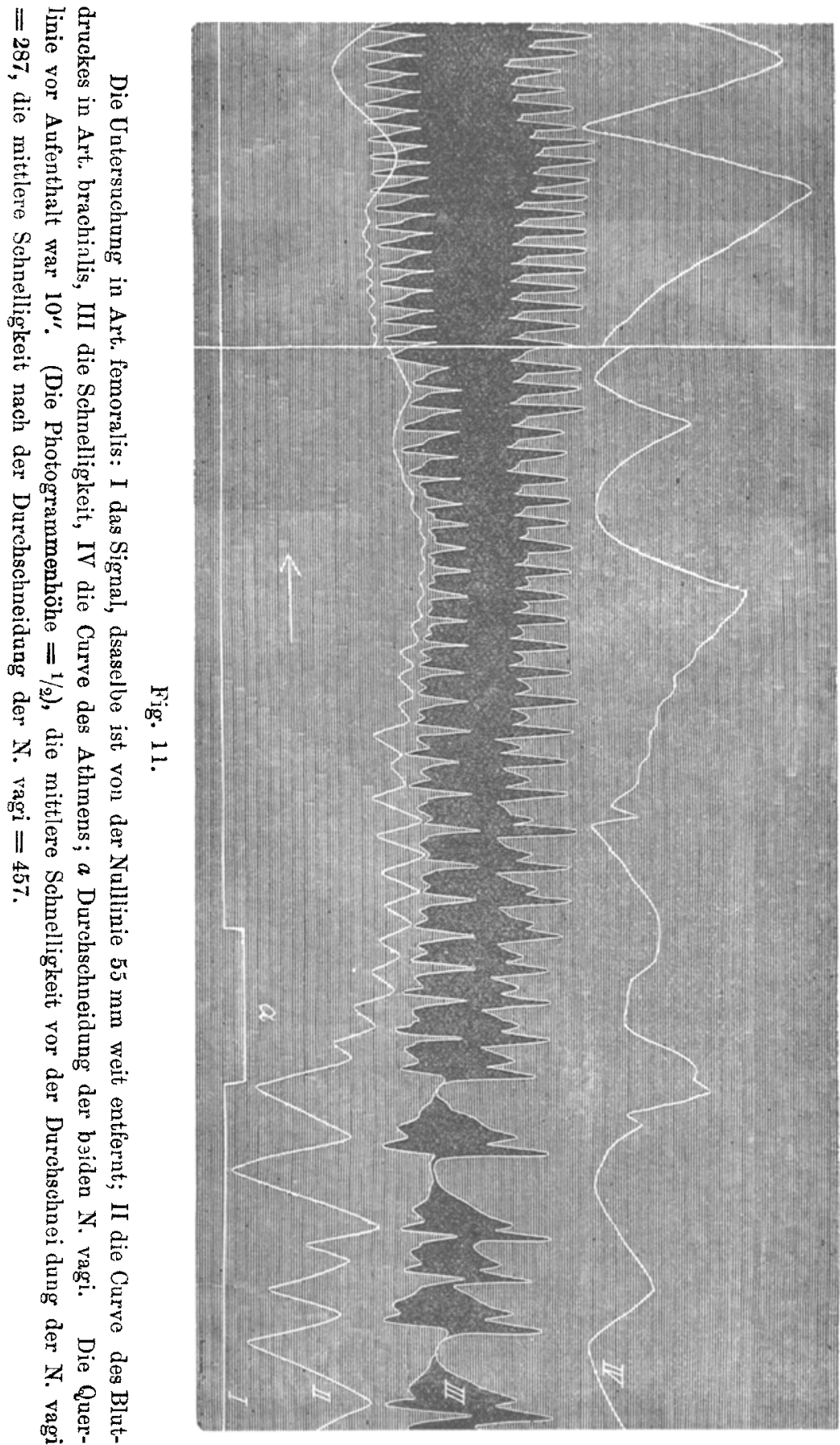

